\title{
Null Kähler Geometry and Isomonodromic Deformations
}

\author{
Maciej Dunajski \\ Department of Applied Mathematics and Theoretical Physics, University of Cambridge, Wilberforce Road, \\ Cambridge CB3 0WA, UK. E-mail: m.dunajski@damtp.cam.ac.uk
}

Received: 16 September 2021 / Accepted: 4 November 2021

Published online: 8 December 2021 - (C) The Author(s) 2021

\section{Dedicated to Jenya Ferapontov on the occasion of his 60th birthday}

\begin{abstract}
We construct the normal forms of null-Kähler metrics: pseudo-Riemannian metrics admitting a compatible parallel nilpotent endomorphism of the tangent bundle. Such metrics are examples of non-Riemannian holonomy reduction, and (in the complexified setting) appear on the space of Bridgeland stability conditions on a CalabiYau threefold. Using twistor methods we show that, in dimension four-where there is a connection with dispersionless integrability - the cohomogeneity-one anti-self-dual null-Kähler metrics are generically characterised by solutions to Painlevé I or Painlevé II ODEs.
\end{abstract}

\section{Introduction}

A null-Kähler structure on a manifold $\mathcal{X}$ of real dimension $4 n$ consists of a pseudoRiemannian metric $g$ of signature $(2 n, 2 n)$, together with a rank $2 n$ endomorphism $N$ of $T \mathcal{X}$ which, for all vector fields $X, Y$, satisfies

$$
g(X, N Y)+g(N X, Y)=0, \quad N^{2}=0
$$

and is parallel with respect to the Levi-Civita connection of $g$.

There are three reasons for considering such structures. Firstly, they provide an example of a pseudo-Riemannian holonomy reduction with no Riemannan analogue $[2,6,10,19]$ (in the sense that a null-Kähler metric can not be analyticaly continued to Riemannian signature). Secondly, there exists a natural fibration of $\mathcal{X}$ ovesr a symplectic manifold of dimension $2 n$, such that the pull-back of the symplectic form to $\mathcal{X}$ agrees with the fundamental form $\Omega$ defined by $\Omega(X, Y)=g(N X, Y)$. This structure, albeit in the complexified setup and under the additional condition that $g$ is hyper-Kähler, underlies the Bridgeland approach to stability conditions on a three-dimensional CalabiYau triangulated category [7-9]. Finally, in dimension four and under the additional anti-self-duality assumption, null-Kähler structures are characterised by solutions to a dispersionless integrable system [15]. 
In the next section we shall introduce null Hermitian structures on vector spaces, and in Sect. 3 we put these structures on manifolds. Our main result in Sect. 3 is the local normal form of the null-Kähler condition

Theorem 1.1. Let $(\mathcal{X}, g, N)$ be a $4 n$-dimensional null-Kähler manifold. There exist a local coordinate system $\left(x^{i}, y^{i}\right), i=1, \ldots, 2 n$ and a function $\Theta: \mathcal{X} \rightarrow \mathbb{R}$ such that

$$
\begin{aligned}
& g=\frac{1}{2} \sum_{i, j} \omega_{i j}\left(d y^{i} \otimes d x^{j}+d x^{j} \otimes d y^{i}\right)+\frac{\partial^{2} \Theta}{\partial y^{i} \partial y^{j}}\left(d x^{i} \otimes d x^{j}+d x^{j} \otimes d x^{i}\right), \\
& N=\sum_{i} d x^{i} \otimes \frac{\partial}{\partial y^{i}}, \quad \text { where } \omega_{i j}=\left(\begin{array}{cc}
0 & \mathbb{I}_{n} \\
-\mathbb{I}_{n} & 0
\end{array}\right) .
\end{aligned}
$$

Conversely, the structure (1.1) is null-Kähler for any function $\Theta=\Theta\left(x^{i}, y^{i}\right)$.

Thus, in the real analytic category, a null-Kähler manifold depends on one arbitrary function of $4 n$ variables. In Sects. 3.2 and 3.3 we shall list systems of PDEs satisfied by this function if the metric is Einstein or (complexified) hyper-Kähler together with examples.

In Sect. 4 we focus on oriented four-dimensional null-Kähler structures, with the natural choice of orientation which makes the fundamental form $\Omega$ self-dual. While null-Kähler metrics with self-dual Weyl tensor can be found explicitly, the anti-selfduality condition on Weyl tensor corresponds to solutions to a 4th order dispersionless integrable system [15]. A problem of finding Ricci-flat null reduces to a non-integrable hyper-heavenly equation of Plebanski and Robinson [30].

In Sect. 5 we establish the main result of the paper. Imposing the invariance under the isometric action of $S L(2)$ on anti-self-dual null-Kähler structures leads to an ODE. By exploring the connection between the twistor distribution and the isomonodromic Lax pair we shall show that this ODE is either completely solvable, or transforms to Painlevé I or Painlevé II.

Theorem 1.2. Let $(\mathcal{X}, g, N)$ be an anti-self-dual null-Kähler four-manifold with an isometric action of $S L(2)$ with three-dimensional orbits, and preserving the endomorphism $N$. Then either the metric $g$ is conformal to a Ricci-flat metric, or it can be put in the form

$$
g=\sum_{\alpha, \beta=1}^{3} \gamma_{\alpha \beta}(t) \sigma^{\alpha} \otimes \sigma^{\beta}+\sum_{\alpha=1}^{3} n_{\alpha}(t)\left(\sigma^{\alpha} \otimes d t+d t \otimes \sigma^{\alpha}\right),
$$

where the function $t: \mathcal{X} \rightarrow \mathbb{R}$ parametrises the orbits of $S L(2)$, and is constant on each orbit, $\left(\sigma^{1}, \sigma^{2}, \sigma^{3}\right)$ are left-invariant one-forms on $S L(2)$ which satisfy (5.6), $\gamma$ is a symmetric 3 by 3 matrix and $n$ is a vector with components given by (5.28), or depending on solutions of Painlevé I or Painlevé II as in (5.27) and (5.25).

Anti-self-dual $S L(2)$ invariant Ricci-flat metrics in neutral signature are all known [17], so the novelty in Theorem 1.2 lies in the apperance of Painlevé equations in the conformal structures with no Ricci-flat representatives. The connection between the first two Painlevé transcendents, and anti-self-dual null-Kähler structures has twistorial origins: the additional structure on the twistor space $\mathcal{Y}$ of $\mathcal{X}$ which corresponds to the endomorophism $N$ is a holomorphic section of $\kappa^{-1 / 4}$, where $\kappa$ is the holomorphic canonical 
bundle of $\mathcal{Y}$. The isometric $S L(2)$ action on $\mathcal{Y}$ also gives rise to such section if the holomorphic vector fields generating this action are linearly dependent at one point, to order four, on each twistor lines. This corresponds to the isomonodromic problem underlying Painlevé I and II.

\section{Algebraic Preliminaries}

Let $V$ be a vector space over field $\mathbb{F}$, where $\mathbb{F}$ is $\mathbb{R}$ or $\mathbb{C}$.

Definition 2.1. A null structure on an even-dimensional vector space $V$ is an endomorphism $N$ of $V$ such that

$$
N^{2}=0
$$

and $\operatorname{rank}(N)=\frac{1}{2} \operatorname{dim}(V)$.

For reasons to become clear later (see Sect. 3) we shall chose $\operatorname{dim}(V)=4 n$, where $n$ is an integer. The kernel of $N$ is a $2 n$-dimensional subspace of $V$, and any basis of this kernel can be extended to a basis of $V$. A convenient way to do it is to pick $2 n$ linearly independent vectors $\left(X_{1}, X_{2}, \ldots, X_{2 n}\right)$ not in $\operatorname{Ker}(N)$, and use

$$
X_{1}, \ldots, X_{2 n}, N\left(X_{1}\right), \ldots, N\left(X_{2 n}\right)
$$

as a basis of $V$. We shall call this basis adapted to $N$. The matrix of $N$ with respect to an adapted basis is

$$
N_{0}=\left(\begin{array}{cc}
0 & \mathbb{I}_{2 n} \\
0 & 0
\end{array}\right),
$$

where $\mathbb{I}_{2 n}$ is the $2 n \times 2 n$ identity matrix.

Let $N$ and $N^{\prime}$ be two null structures on vector spaces $V$ and $V^{\prime}$ respectively. A linear map $\phi: V \rightarrow V^{\prime}$ is called null linear if

$$
N^{\prime} \circ \phi=\phi \circ N \text {. }
$$

The sub-group $\mathcal{N}(V) \subset G L(V)$ of null-linear maps consists of matrices which commute with the matrix $N_{0}$. These matrices are of the form

$$
\left(\begin{array}{ll}
A & B \\
0 & A
\end{array}\right)
$$

where $A$ and $B$ is an arbitrary $2 n \times 2 n$ matrix over $\mathbb{F}$, and $A$ is invertible.

Proposition 2.2. There is a one-to-one correspondence between the set of null structures on $V$, and the homogeneous space $G L(V) / \mathcal{N}(V)$.

Proof. Consider a $G L(V)$ action of a space of null structures given by

$$
N \longrightarrow \phi N \phi^{-1}, \quad \phi \in G L(V) \text {. }
$$

Let $N$ and $N^{\prime}$ be two null structures of $V$ with adapted bases $\left(X_{i}, N\left(X_{i}\right)\right.$ and $\left(X^{\prime}{ }_{i}, N\left(X^{\prime}{ }_{i}\right)\right)$ respectively, where $i=1, \ldots, 2 n$. Define the element $\phi \in G L(V)$ by

$$
\phi\left(X_{i}\right)=X_{i}^{\prime}, \quad \phi\left(N\left(X_{i}\right)\right)=N^{\prime}\left(X_{i}^{\prime}\right) .
$$

Therefore $N^{\prime}=\phi N \phi^{-1}$ and the action (2.3) is transitive. The isotropy subgroup is of this action is $\mathcal{N}(V)$, as $N_{0}=\phi N_{0} \phi^{-1}$ iff $\phi \in \mathcal{N}(V)$. 
Our elementary discussion has so far followed the standard treatment of complex structures [23], except the endomorphism $N$ squares to 0 rather than $-\mathbb{I}_{4 n}$. The next step is to introduce the analog of Hermitian inner products ${ }^{1}$

Definition 2.3. A non-degenerate symmetric bi-linear inner product $g: V \times V \rightarrow \mathbb{F}$ is called null-Hermitian if

$$
g(N X, Y)=-g(X, N Y),
$$

for all $X, Y \in V$, where $N$ is a null structure of $V$.

If $\mathbb{F}=\mathbb{R}$, then the signature of a null-Hermitian inner product is $(2 n, 2 n)$, also called split, neutral or Kleinian. The definition 2.3 also implies $g(X, N X)=0$ and that $\operatorname{Ker}(N)$ is a totally isotropic subspace of $V$.

To each null-Hermitian inner product we associate a skew-symmetric bi-linear map $\Omega \in \Lambda^{2}\left(V^{*}\right)$ defined by

$$
\Omega(X, Y)=g(N X, Y) .
$$

Therefore $\Omega$ vanishes on $\operatorname{Ker}(N)$, and it equips the $2 n$-dimensional quotient vector space $V / \operatorname{Ker}(N)$ with a symplectic structure. In a basis adapted to $N$ the inner product $g$ and the skew-form $\Omega$ are represented by

$$
g_{0}=\left(\begin{array}{cc}
0 & \omega \\
\omega^{T} & 0
\end{array}\right), \quad \Omega_{0}=\left(\begin{array}{cc}
\omega & 0 \\
0 & 0
\end{array}\right), \quad \text { where } \omega=\left(\begin{array}{cc}
0 & \mathbb{I}_{n} \\
-\mathbb{I}_{n} & 0
\end{array}\right) .
$$

Example 2.1. Let $W$ be a $2 n$-dimensional symplectic vector space with a symplectic form $\omega$. The $4 n$-dimensional space $V=W \oplus W$ carries a null-Hermitian structure defined by

$$
N(x, y)=(0, x), \quad g\left((x, y),\left(x^{\prime}, y^{\prime}\right)\right)=\omega\left(x, y^{\prime}\right)-\omega\left(y, x^{\prime}\right)
$$

where $\left(x, y, x^{\prime}, y^{\prime}\right) \in W$. This inner product has signature $(2 n, 2 n)$ and it indeed satisfies (2.4) as

$$
g\left((x, y), N\left(x^{\prime}, y^{\prime}\right)\right)=g\left(\left(0, x^{\prime}\right),(x, y)\right)=\omega\left(x^{\prime}, x\right)=-g\left(N(x, y),\left(x^{\prime}, y^{\prime}\right)\right) .
$$

1 The natural next step in the theory of complex structures is to introduce a complexification, where multiplication by complex numbers is given in terms of the complex structure $J$ by $(a+i b) X=a+b J(X)$, where $X \in V, a, b \in \mathbb{R}$ and $i^{2}=-1$. Pursuing this analogy for null structures leads to dual numbers in place of complex numbers. The algebra $\mathbb{D}$ of dual numbers consists of elements of the form

$$
a+\epsilon b, \text { where } a, b \in \mathbb{R} \text { and } \epsilon^{2}=0 .
$$

The dual numbers can be added, and multiplied according to

$$
\left(a_{1}+\epsilon b_{1}\right)\left(a_{2}+\epsilon b_{2}\right)=a_{1} a_{2}+\epsilon\left(a_{1} b_{2}+b_{1} a_{2}\right), \quad\left(a_{1}+\epsilon b_{1}\right)+\left(a_{2}+\epsilon b_{2}\right)=a_{1}+a_{2}+\epsilon\left(b_{1}+b_{2}\right),
$$

but $\mathbb{D}$ is not a division algebra, as elements of the form $\epsilon b$ do not have inverses. The infinitesimal dual number $\epsilon$ underlies non-standard analysis, as it gives a framework to distinguish between real numbers like 1 , and $0.999 \ldots$ which are regarded as equal in ordinary analysis.

A real vector space $V$ with a null structure $N$ can be turned into a vector space $V^{\mathbb{D}}$ over $\mathbb{D}$ by defining

$$
(a+\epsilon b) X=a X+b N(X), \text { where } X \in V \quad \text { and } a, b \in \mathbb{R} .
$$

In what follows we shall not explore any further connection between null structures and dual numbers, but will instead focus on null structures on curved manifolds. 
Example 2.2. Let $V$ be a $4 n$-dimensional vector space over $\mathbb{F}=\mathbb{R}$ with two null structures $N_{1}, N_{2}$, such that

$$
N_{1} N_{2}+N_{2} N_{1}=-\mathrm{Id},
$$

where Id is the identity endomorphism on $V$. Then the endomorphisms

$$
I:=N_{1}+N_{2}, \quad S:=N_{1}-N_{2}, \quad T:=\left[N_{1}, N_{2}\right]
$$

equip $V$ with a pseudo-quaternionic structure. Indeed

$$
\begin{aligned}
& I^{2}=N_{1} N_{2}+N_{2} N_{1}=-\mathrm{Id}, \\
& S^{2}=-N_{1} N_{2}-N_{2} N_{1}=\mathrm{Id}, \\
& T^{2}=N_{2}\left(-\mathrm{Id}-N_{2} N_{1}\right) N_{1}+N_{1}\left(-\mathrm{Id}-N_{1} N_{2}\right) N_{2}=\mathrm{Id}, \\
& I S=-T=-S I, \quad I T=S=-T I, \quad S T=I=-T S .
\end{aligned}
$$

If we instead consider $V$ over $\mathbb{F}=\mathbb{C}$ then $I, J:=i S, K:=-i T$ form a complexified hyper-complex structure on $V$. Then $\frac{1}{2}(I \pm i J)$ are null structures on $V$.

\section{Null Kähler Structures}

Let $\mathcal{X}$ be a smooth manifold of real dimension $4 n$. We shall equip $\mathcal{X}$ with a null structure by smoothly extending such structure from each tangent space, and imposing integrability conditions.

Definition 3.1. A null structure $N$ on $\mathcal{X}$ is an endomorphism $N: T \mathcal{X} \rightarrow T \mathcal{X}$ such that $N^{2}=0$, and the sub-bundle $\mathcal{D} \subset T \mathcal{X}$ consisting of vectors fields annihilated by $N$ has rank $2 n$, and is Frobenius-integrable, i. e. $[\mathcal{D}, \mathcal{D}] \subset \mathcal{D}$.

The integrability condition holds if $N[N X, N Y]=0$ or equivalently if $T(N X, Y)=0$ for all vector fields $X, Y$, where

$$
T(X, Y):=[N X, N Y]-N[N X, Y]-N[X, N Y]
$$

is the Nijenhuis tensor of $N$.

Null-structures are also called almost-tangent structures [37], and the following example shows why

Example 3.1. Let $\mathcal{X}=T M$ be the total space of the tangent bundle to a $2 n$ dimensional manifold $M$. Let $U \in \Gamma(T M)$ be a vector field on $M$. Recall [38] that the vertical lift $U^{V}$ of $U$ to $T M$ is a section of $T(T M)$ defined by

$$
U^{V}(f)=\left.\frac{d}{d \epsilon}\right|_{\epsilon=0} f(m, u+\epsilon U)
$$

where $f: T M \rightarrow \mathbb{R}$ is an arbitrary function, $m \in M$ and $u \in T_{m} M$. The canonical null structure on $T M$ is the endomorphism $N: T(T M) \rightarrow T(T M)$ defined by

$$
N(X)=\left[\pi_{*}(X)\right]^{V},
$$

where $\pi_{*}$ is the tangent map to the bundle projection $\pi: T M \rightarrow M$. 
Let $\left(x^{1}, \ldots, x^{2 n}\right)$ be local coordinates on $M$ covering a neighbourhood of $m \in M$, and $\left(y^{1}, \ldots, y^{2 n}\right)$ be the natural coordinates on $T_{m} M$ obtained by writing a tangentvector as $U=\sum_{i} y^{i} \frac{\partial}{\partial x^{i}}$. Then $\left(x^{i}, y^{i}\right)$ are local coordinates on $T M$. If

$$
X=\sum_{i} A^{i} \frac{\partial}{\partial x^{i}}+B^{i} \frac{\partial}{\partial y^{i}}
$$

is a general vector field on $T M$, then (3.1) implies

$$
N(X)=\sum_{i} A^{i} \frac{\partial}{\partial y^{i}} .
$$

Thus, in the natural coordinate system $\left(x^{i}, y^{i}\right)$ on $T M$ the null structure is given by a $(1,1)$ tensor $^{2}$

$$
N=\sum_{i} d x^{i} \otimes \frac{\partial}{\partial y^{i}}
$$

Definition 3.2. A signature $(2 n, 2 n)$ pseudo-Riemannian metric $g$ on a manifold $\mathcal{X}$ with a null structure $N$ is called null-Kähler if

$$
g(N X, Y)=-g(X, N Y) \quad \text { and } \quad \nabla N=0
$$

where $\nabla$ is the Levi-Civita connection of $g$.

The fundamental two-form $\Omega \in \Lambda^{2}\left(T^{*} \mathcal{X}\right)$ defined by

$$
\Omega(X, Y)=g(N X, Y)
$$

is covariantly-constant, and therefore closed. It satisfies

$$
\Omega^{\wedge n}:=\underbrace{\Omega \wedge \cdots \wedge \Omega}_{n} \neq 0, \quad \Omega^{\wedge(n+1)}=0 .
$$

This should be contrasted with the Kähler condition, where $\Omega^{\wedge 2 n} \neq 0$, and justifies the terminology.

Example 3.2. Let $\mathcal{X}_{\mathbb{C}}$ be a complexified hyper-Kähler manifold, i.e. a complex manifold of complex dimension $4 n$ with three holomorphic parallel complex structures $I, J, K$ satisfying the algebra of quaternions and Hermitian with respect to a holomorphic metric $g$ on $\mathcal{X}_{\mathbb{C}}$. Then $N=\frac{1}{2}(I+i J)$ is a (one of many) null-Kähler structure on $\mathcal{X}_{\mathbb{C}}$. This example underlies the occurrence of null structures in the geometric approach to Donaldson-Thomas invariants [7,9].

In the Sect. 3.1 we shall present a canonical normal-form of null-Kähler metrics. In the rest of this section we list properties of such metrics which do not refer to any choices of coordinates.

\footnotetext{
2 There is another, equivalent definition of this canonical null structure which we shall now describe. We have defined vertical lifts of vector fields to the tangent bundle. We can also define vertical lifts of functions: if $h: M \rightarrow \mathbb{R}$, then $h^{V}=h \circ \pi$ is a function on $T M$. Vertical lifts of all tensor fields are defined by $(P \otimes Q)^{V}=P^{V} \otimes Q^{V}$. In particular the vertical lift of the identity endomorphism of $T M$ to $T(T M)$ is given by $N$.
} 
Proposition 3.3. The Riemann curvature R, the Ricci curvature $r$ and the Ricci scalar $S$ of a null-Kähler metric satisfy

$$
\begin{aligned}
R(X, Y) \circ N & =N \circ R(X, Y), \\
R(N X, Y) & =-R(X, N Y), \\
r(N X, Y) & =0, \\
S & =0 .
\end{aligned}
$$

Proof. Formula (3.3a) follows directly from $\nabla N=0$, and the definition of the curvature

$$
R(X, Y) V=\left[\nabla_{X}, \nabla_{Y}\right] V-\nabla_{[X, Y]} V .
$$

To prove (3.3b) we use the (3.3a) together with the symmetry properties of the Riemannian curvature:

$$
\begin{aligned}
g(R(N X, Y) V, U) & =g(R(U, V) Y, N X)=-g(N R(U, V) Y, X) \\
& =-g(R(U, V) N Y, X)=-g(R(X, N Y) V, U) .
\end{aligned}
$$

From its definition

$$
r(X, Y)=\operatorname{Tr}(V \rightarrow R(V, X) Y)
$$

The third formula (3.3c) then follows if we take $V \in \mathcal{D}$ as, setting $V=N U$, we have

$$
\begin{aligned}
r(N X, Y) & =\operatorname{Tr}(N U \rightarrow R(N U, N X) Y) \\
& =-\operatorname{Tr}\left(N U \rightarrow R\left(U, N^{2} X\right) Y\right)=0 .
\end{aligned}
$$

Finally, to prove (3.3d) we shall compute $S=\operatorname{Tr}_{g}(r)$ in the basis adapted to $N$, and regard $r$ and $g^{-1}$ as linear maps. The property (3.3c) implies that in this basis the matrix of $r$ is of the form

$$
\left(\begin{array}{ll}
* & 0 \\
0 & 0
\end{array}\right) .
$$

The property (2.4) implies that the matrix of $g^{-1}$ is of the form

$$
\left(\begin{array}{cc}
0 & \omega \\
\omega^{T} & \Theta
\end{array}\right) .
$$

for some block $2 n$ by $2 n$ matrices $\Theta$ and $\omega$ such that $\omega$ is skew and non-degenerate. Therefore $S=\operatorname{Tr}\left(g^{-1} \cdot r\right)=0$.

The next result relates null-Kähler structures to special holonomy, and manifolds with parallel spinors $[2,10]$

Proposition 3.4. A null-Kähler manifold admits a canonical parallel pure spinor. 
Proof. This is really a result in linear algebra which builds on a bijection between the set of pure semi-spinors in $V=\mathbb{R}^{2 n, 2 n}$ and the Grasmannian of totally null $2 n$-dimensional subspaces of $V$. Let $\mathcal{C} l(2 n, 2 n)$ be a real $2^{4 n}$-dimensional Clifford algebra (see, e. g. [24]) of a null-Hermitian space $\left(V=\mathbb{R}^{2 n, 2 n}, N\right)$. This algebra is generated by $2^{2 n} \times 2^{2 n}$ matrices $\gamma(X)$ subject to the relations

$$
\gamma(X) \gamma(Y)+\gamma(Y) \gamma(X)=2 g(X, Y) \mathbf{1}, \quad \text { where } X, Y \in V .
$$

The multiplicative group $\operatorname{Spin}(2 n, 2 n)$ is generated by all elements $\gamma(X) \gamma(Y)$, where $X, Y$ are vectors of squared norm \pm 1 . The spin space $\mathbb{S}$ is a reducible representation space of $\operatorname{Spin}(2 n, 2 n)$. It can be decomposed as

$$
\mathbb{S}=\mathbb{S}_{+} \oplus \mathbb{S}_{-} \cong \mathbb{R}^{2^{2 n-1}} \oplus \mathbb{R}^{2^{2 n-1}}
$$

where $\mathbb{S}_{ \pm}$are irreducible spaces of semi-spinors. It is a simple algebraic fact that any totally isotropic subspace of $V$ has dimension at most $2 n$. A semi-spinor $\iota$ is called pure iff

$$
\operatorname{dim}\{X \in V, \gamma(X) \iota=0\}=2 n
$$

The system of equations underlying (3.4) is of rank $2 n$, and defines $2 n$-dimensional plane. This plane is totally isotropic as

$$
0=\gamma(X) \gamma(X) \iota=g(X, X) \iota
$$

so $g(X, X)=0$. The space of totally isotropic planes in $\mathbb{R}^{2 n, 2 n}$ has two components defined by a pure element of $\mathbb{S}_{+}$and $\mathbb{S}_{-}$respectively. A pure semi-spinor $\iota$ is annihilated by $\gamma\left(X_{1}\right) \gamma\left(X_{2}\right) \cdots \gamma\left(X_{2 n}\right) \in \mathcal{C} l(2 n, 2 n)$ where $X_{1}, \cdots, X_{2 n}$ span a totally null plane. Therefore $\iota$ corresponds to an element of the Grassmann algebra $\xi \in \Lambda^{2 n}\left(V^{*}\right)$ such that $\xi \wedge \xi=0$, and the assertion of the Proposition follows because $\xi$ is defined by a null-Hermitian structure $\xi=\Omega^{\wedge n}$, and therefore is parallel.

If $n=1$ then all semi-spinors are pure. The first non-trivial case corresponds to 8-dimensional null-Hermitian structures.

3.1. Null Kähler Potential. In this section we shall find a canonical normal form of a null-Kähler metric, and express it in terms of second derivatives of one arbitrary function on $\mathcal{X}$.

Proof of Theorem 1.1. Let

$$
M=\mathcal{X} / \operatorname{Ker}(N)
$$

be a $2 n$-dimensional quotient manifold by the kernel of the $2 n$-dimensional integrable distribution $\mathcal{D}$ of vector fields annihilated by $N$. Locally we regard $\mathcal{X}$ is the tangent bundle to $M$, and in the coordinate system of Example 3.1 the endomorphism $N$ is

$$
N=\sum_{i} d x^{i} \otimes \frac{\partial}{\partial y^{i}},
$$


where $\left(x^{1}, \ldots, x^{2 n}\right)$ are local coordinates on $M$, and $\left(y^{1}, \ldots, y^{2 n}\right)$ are linear coordinates on fibres of $T M \rightarrow M$. The distribution $\mathcal{D}=\operatorname{span}\left\{\partial / \partial y^{1}, \ldots, \partial / \partial y^{2 n}\right\}$ is totally isotropic, and therefore there exists a collection of functions on $\mathcal{X}$

$$
\omega_{i j}=\omega_{i j}(x, y), \quad \Theta_{i j}=\Theta_{i j}(x, y)
$$

such that $\Theta_{i j}=\Theta_{j i}$ and

$$
g=\frac{1}{2} \sum_{i, j} \omega_{i j}\left(d y^{i} \otimes d x^{j}+d x^{j} \otimes d y^{i}\right)+\frac{1}{2} \sum_{i, j} \Theta_{i j}\left(d x^{i} \otimes d x^{j}+d x^{j} \otimes d x^{i}\right)
$$

Evaluating the null-Kähler condition (3.2) on coordinate vector fields shows that

$$
\omega_{i j}=-\omega_{j i}
$$

We now impose the parallel condition $\nabla N=0$, where $N$ is given by (3.5). The $d y^{i} \otimes d x^{j}$ components of $\nabla N$ vanish iff

$$
\frac{\partial \omega_{i j}}{\partial y^{k}}=0
$$

so that $\omega_{i j}=\omega_{i j}(x)$, and

$$
\omega=\frac{1}{2} \sum_{i, j} \omega_{i j} d x^{i} \wedge d x^{j}
$$

is a symplectic form on $M$. Locally there exists a diffeomorphism of $M$ to a Darboux coordinate system $\tilde{x}=\tilde{x}(x)$ such that

$$
\omega=d \tilde{x}^{1} \wedge d \tilde{x}^{n+1}+\cdots+d \tilde{x}^{n} \wedge d \tilde{x}^{2 n} .
$$

The transformation of $\mathcal{X}=T M$ induced by this diffeomorphism is

$$
\tilde{x}^{i}=\tilde{x}^{i}\left(x^{j}\right), \quad \tilde{y}^{i}=\sum_{j} \frac{\partial \tilde{x}^{i}}{\partial x^{j}} y^{j}, \quad i, j=1, \ldots, 2 n,
$$

and it preserves the form of $N$, as $N=\sum_{i} d \tilde{x}^{i} \otimes \partial / \partial \tilde{y}^{i}$. We shall use this Darboux coordinate system from now on, and drop tildes. This has an effect of reducing $\omega_{i j}$ to a constant symplectic matrix as in (1.1). We now move on the vanishing of $d x^{i} \otimes d x^{j}$ components in $\nabla N$. This is equivalent to

$$
\frac{\partial \Theta_{i j}}{\partial y^{k}}=\frac{\partial \Theta_{i k}}{\partial y^{j}}
$$

which gives the integrability conditions for the existence of $2 n$ function $\Theta_{1}, \ldots, \Theta_{2 n}$ on $\mathcal{X}$ such that

$$
\Theta_{i j}=\frac{\partial \Theta_{i}}{\partial x^{j}}
$$

The symmetry condition $\Theta_{i j}=\Theta_{j i}$ implies the existence of a single function $\Theta=$ $\Theta(x, y)$ such that

$$
\Theta_{i}=\frac{\partial \Theta}{\partial y^{i}} .
$$

This puts the metric (3.6) in the canonical form (1.1). 
The local normal form (1.1) is not invariant under general diffeomorphisms of $\mathcal{X}$. The subgroup of the pseudogroup of all diffeomorphisms changing coordinates $\left(x^{i}, y^{i}\right)$ as well as $\Theta$, while preseving (1.1) is a semi-direct product of SDiff(M) and $\Gamma(M)$, where the symplectomorphisms $\operatorname{SDiff}(M)$ of $M$ act on $\mathcal{X}=T M$ by a Lie lift, and $\Gamma(M)$ acts on the fibres of $T M$ by translations. The details are as follows: Let $Y$ be a vector field on $\mathcal{X}$ generating a one-parameter group of diffeomorphisms. The conditions

$$
\mathcal{L}_{Y} \Omega=0, \quad \mathcal{L}_{Y} N=0
$$

imply

$$
Y=\sum_{i, j} \omega^{i j} \frac{\partial H}{\partial x^{j}} \frac{\partial}{\partial x^{i}}+\sum_{i, j, k}\left(y^{k} \omega^{i j} \frac{\partial^{2} H}{\partial x^{k} \partial x^{j}}\right)+\sum_{i} T^{i} \frac{\partial}{\partial y^{i}},
$$

where $\omega^{i j}$ is the inverse matrix of $\omega_{i j}$, i. e. $\omega^{i k} \omega_{k j}=\delta^{i}{ }_{j}$, and $\left(H, T^{1}, \ldots, T^{2 n}\right)$ are arbitrary functions of $\left(x^{1}, \ldots, x^{2 n}\right)$. Set

$$
\tilde{x}^{i}=x^{i}+\epsilon \mathcal{L}_{Y}\left(x^{i}\right), \quad \tilde{y}^{i}=y^{i}+\epsilon \mathcal{L}_{Y}\left(y^{i}\right), \quad \widetilde{\Theta}=\Theta+\epsilon \delta \Theta .
$$

Using

$$
\frac{\partial}{\partial \tilde{y}^{i}}=\frac{\partial}{\partial y^{i}}-\sum_{j, k} \epsilon \omega^{j k} \frac{\partial^{2} H}{\partial y^{i} \partial y^{k}} \frac{\partial}{\partial y^{j}}
$$

we find that the action generated by $Y$ preserves the form of $g$, i. e.

$g=\frac{1}{2} \sum_{i, j} \omega_{i j}\left(d \tilde{y}^{i} \otimes d \tilde{x}^{j}+d \tilde{x}^{j} \otimes d \tilde{y}^{i}\right)+\frac{\partial^{2} \widetilde{\Theta}}{\partial \tilde{y}^{i} \partial \tilde{y}^{j}}\left(d \tilde{x}^{i} \otimes d \tilde{x}^{j}+d \tilde{x}^{j} \otimes d \tilde{x}^{i}\right)+O\left(\epsilon^{2}\right)$

if

$$
\delta \Theta=\sum_{i, j, k}\left(\frac{1}{6} y^{i} y^{j} y^{k} \frac{\partial^{3} H}{\partial x^{i} \partial x^{j} \partial x^{k}}-\frac{1}{2} y^{j} y^{k} \omega_{i j} \frac{\partial T^{i}}{\partial x^{k}}\right)+\sum_{i} y^{i} Q_{i}+R
$$

where $\left(Q_{1}, \ldots, Q_{2 n}, R\right)$ are arbitrary functions of $x^{i}$.

3.2. Null-Kähler Einstein Metrics. Computing the Ricci tensor of a null-Kähler metric in the form (1.1) we find

$$
r=\sum_{i, j} \frac{\partial^{2} f}{\partial y^{i} \partial y^{j}}\left(d x^{i} \otimes d x^{j}+d x^{j} \otimes d x^{i}\right)
$$

where

$$
f \equiv \sum_{i, j} \omega^{i j} \frac{\partial^{2} \Theta}{\partial y^{i} \partial x^{j}}+\sum_{i, j, k, l} \frac{1}{2} \omega^{i k} \omega^{j l} \frac{\partial^{2} \Theta}{\partial y^{i} \partial y^{j}} \frac{\partial^{2} \Theta}{\partial y^{k} \partial y^{l}} .
$$


The Ricci-flat condition on $g$ therefore reduces to a system of fourth order PDEs on $\Theta$ which can be integrated twice to give a single second orde PDE on $\Theta$

$$
f=G+y^{i} F_{i}
$$

where $\left(G, F_{1}, \ldots, F_{2 n}\right)$ are arbitrary functions of $x^{i}$. Applying the Cauchy-Kovalevskaya theorem shows that in the real-analytic category the general Ricci-flat null Kähler metric depends on two arbitrary functions of $4 n-1$ variables and some number of functions of $2 n$ variables.

Example 3.3. It can be explicitly verified that for

$$
\Theta=\frac{c}{\rho^{2 n-1}} \quad \text { where } \rho=\sum_{i, j} \omega_{i j} y^{i} x^{j}, \quad c=\text { const }
$$

the linear and non-linear terms in (3.8) vanish separately resulting in $f=0$. The resulting metric

$$
g=\frac{1}{2} \sum_{i, j} \omega_{i j}\left(d y^{i} \otimes d x^{j}+d x^{j} \otimes d y^{i}\right)+\frac{2 c n(2 n-1)}{\rho^{2 n+1}}\left(\sum_{k, l} \omega_{k l} x^{k} d x^{l}\right)^{\otimes 2}
$$

is therefore null-Kähler, and Ricci-flat. This metric with $n=1$ is the Sparling-Tod $H$-space [31].

3.3. Complex Hyper-Kähler Metrics with Affine Symplectic Fibrations. In the complexified setting the coordinates $\left(x^{i}, y^{i}\right)$ are holomorphic on the complex manifold $\mathcal{X}_{\mathbb{C}}$ of complex dimension $4 n$. If $\Theta=\Theta(x, y)$ satisfies the system of PDEs

$$
\begin{aligned}
& H_{i j}=0, \quad i, j=1, \ldots, 2 n \text { where } \\
& H_{i j} \equiv \frac{\partial^{2} \Theta}{\partial y^{i} \partial x^{j}}-\frac{\partial^{2} \Theta}{\partial y^{j} \partial x^{i}}+\sum_{k, l} \omega^{k l} \frac{\partial^{2} \Theta}{\partial y^{i} \partial y^{l}} \frac{\partial^{2} \Theta}{\partial y^{j} \partial y^{k}}=0,
\end{aligned}
$$

then the metric (1.1) is complexified hyper-Kähler. In [9] it was shown that if $\left(M_{\mathbb{C}}, \omega\right)$ is a complexifed symplectic manifold of complex dimension $2 n$, and $g$ is a complexified hyper-Kähler metric on $\mathcal{X}_{\mathbb{C}}=T M_{\mathbb{C}}$ such that the null-Kähler two-form

$$
\pi^{*}(\omega)=\Omega_{I}+i \Omega_{J},
$$

then $g$ is locally of the form (1.1), where $\Theta$ satisfies the system (3.11).

The system (3.11) consists of some of the flows of the hyper-Kähler hierarchy [14,32]. It implies the Frobenius integrability

$$
\left[l_{i}, l_{j}\right]=0, \quad i, j=1, \ldots, 2 n
$$

for the rank- $2 n$ distribution spanned by

$$
l_{i}=\frac{\partial}{\partial y^{i}}+\lambda\left(\frac{\partial}{\partial x^{i}}+\sum_{j, k} \omega^{j k} \frac{\partial^{2} \Theta}{\partial y^{i} \partial y^{j}} \frac{\partial}{\partial y^{k}}\right)
$$

on $\mathcal{X}_{\mathbb{C}} \times \mathbb{C P}^{1}$, where $\lambda$ is the affine coordinate on $\mathbb{C P}^{1}$. 
Vanishing of the Lie brackets (3.12) gives a weaker set of conditions ${ }^{3}$

$$
\frac{\partial H_{i j}}{\partial y^{k}}=0, \quad \text { so that } H_{i j}=C_{i j}(x),
$$

for some skew $C_{i j}$. If $n=1$ then a transformation $\Theta \rightarrow \Theta+\sum_{i} y^{i} Q_{i}(x)$ can be used to set $C_{i j}$ to zero. For general $n$ this only seems possible if the two-form $\sum_{i, j} C_{i j} d x^{i} \wedge d x^{j}$ is closed. In [9] it has been argued that for any $n$ the conditions (3.13) together with the additional assumption that the function $\Theta$ is odd in the fibre variables $\left(y^{1}, \ldots, y^{2 n}\right)$ imply (3.11).

Geometrically, $\lambda$ labels the $2 n$-dimensional surfaces (the $\alpha$-surfaces in the twistor approach [14]) through each point of $\mathcal{X}_{\mathbb{C}}$. The twistor space of $\left(\mathcal{X}_{\mathbb{C}}, g\right)$ is the space of these $\alpha$-surfaces. It is a complex manifold of complex dimension $2 n+1$, which arises as the quotient of $\mathcal{X}_{\mathbb{C}} \times \mathbb{C P}^{1}$ by the distribution spanned by $l_{i}$. The points in $\mathcal{X}_{\mathbb{C}}$ correspond to rational curves in $\mathcal{Y}$ with normal bundle $\mathbb{C}^{2 n} \otimes \mathcal{O}(1)$, where $\mathcal{O}(1)$ is the line bundle with Chern class 1 on $\mathbb{C P}^{1}$. In [14] this twistor correspondence has been extended to the full hyper-Kähler hierarchy.

Example 3.4. A strong Joyce structure has, in [8], been defined to be a solution $\Theta$ of the system (3.11) subject to three additional conditions:

(1) $\Theta$ is odd in the variables $y^{i}$.

(2) $Z \equiv \sum_{i} x^{i} \frac{\partial}{\partial x^{i}}$ is a homothetic Killing vector field such that

$$
\mathcal{L}_{Z} g=g, \quad \mathcal{L}_{Z} \Theta=-\Theta .
$$

(3) The metric is invariant under the lattice transformations

$$
y^{i} \rightarrow y^{i}+2 \pi \sqrt{-1}, \quad i=1, \ldots, 2 n .
$$

An example of a solution to (3.11) which also satisfies the three conditions above is

$$
\Theta=\frac{\sinh y^{1}}{x^{1}}
$$

The resulting metric is non-flat, and is an example of a Ricci-flat plane wave.

3.4. Conformal invariance. In four dimension the restricted conformal transformations, where the conformal factor is constant along the distribution $\mathcal{D}=\operatorname{Ker}(N)$, preserve the null-Kähler condition: If $F=F\left(x^{1}, x^{2}\right)$, and

$$
\hat{g}=F^{2} g, \quad \hat{\Omega}=F^{3} \Omega, \quad \text { then } \hat{\nabla} \hat{\Omega}=0 .
$$

This conformal invariance is not present in other dimensions: for $F^{k} \Omega$ to be closed we need $k=0$, and then $\hat{\nabla} \Omega=0$ implies $F=$ const.

\footnotetext{
3 Note that $\sum_{i, j} \omega^{i j} H_{i j}=2 f$. Therefore (3.13) implies Ricci-flatness, but the converse is not true.
} 
3.5. Walker structures. Recall that a distribution $\mathcal{D}$ on a pseudo-Riemannian manifold $\mathcal{X}$ is called parallel if $\nabla_{Y} X \in \Gamma(\mathcal{D})$ for all $X \in \Gamma(\mathcal{D})$ and $Y \in \Gamma(T \mathcal{X})$. The pseudoRiemannian manifolds admitting a parallel distribution of rank equal to half of the manifold dimension are called the Walker manifolds [5], and it was shown by Walker [36], that locally a Walker metric is of the form (3.6) for some functions $\Theta_{i j}$. The nullKähler manifolds form a subclass of the Walker manifolds, where $\Theta_{i j}$ is a Hessian of one function. Indeed, any vector field in $\mathcal{D}$ is of the form $N(X)$ for some $X \in \Gamma(T \mathcal{X})$, and we have

$$
\nabla_{Y} N(X)=N \nabla_{Y} X \in \operatorname{Ker}(N)
$$

\section{Four Dimensions}

The author has first came across the null-Kähler structures when investigating twistor theory and integrability of a certain fourth order PDE in four dimensions [15]. In four dimensions the existence of a maximal rank parallel endomorphism $N$ with $N^{2}=0$ is equivalent to existence of a parallel semi-spinor, i. e. a parallel section of a rank-two symplectic vector bundle which we chose to be $\mathbb{S}_{+} \rightarrow \mathcal{X}$ where

$$
T \mathcal{X} \cong \mathbb{S}_{+} \otimes \mathbb{S}_{-}
$$

is a canonical bundle isomorphism, and $\mathbb{S}_{-}$is another rank-2 symplectic vector bundle. This isomorphism is related to the metric on $\mathcal{X}$ by

$$
g\left(v_{1} \otimes w_{1}, v_{2} \otimes w_{2}\right)=\varepsilon_{+}\left(v_{1}, v_{2}\right) \varepsilon_{-}\left(w_{1}, w_{2}\right)
$$

where $v_{1}, v_{2} \in \Gamma\left(\mathbb{S}_{+}\right)$and $w_{1}, w_{2} \in \Gamma\left(\mathbb{S}_{-}\right)$, and $\varepsilon_{ \pm}$are symplectic structures on $\mathbb{S}_{ \pm}$ which are parallel with respect to $\nabla$. The Hodge $*$ operator is an involution on two-forms, and induces a decomposition

$$
\Lambda^{2}\left(T^{*} \mathcal{X}\right)=\Lambda_{+}^{2}\left(T^{*} \mathcal{X}\right) \oplus \Lambda_{-}^{2}\left(T^{*} \mathcal{X}\right)
$$

of two-forms into self-dual (SD) and anti-self-dual (ASD) components. Given a parallel section of $\mathbb{S}_{+}$, another isomorphism

$$
\Lambda_{+}^{2} \cong \operatorname{Sym}^{2}\left(\mathbb{S}_{+}^{*}\right)
$$

implies the existence of a parallel self-dual two-form $\Omega$ such that $\Omega \wedge \Omega=0$. This two-form, together with $g$ define the nilpotent endomorphism $N$ by (2.5).

In four dimensions there are three non-linear systems of PDEs, one of them completely solvable, one integrable, and one not-integrable, which can be imposed on the null structure. Before writing these systems down in coordinates of Theorem (1.1) recall [1] that in four dimensions the Riemann tensor of $g$ can be regarded as a map $\mathcal{R}: \Lambda^{2}\left(T^{*} \mathcal{X}\right) \rightarrow \Lambda^{2}\left(T^{*} \mathcal{X}\right)$ which admits a decomposition under the splitting (4.2):

$$
\mathcal{R}=\left(\begin{array}{c|c}
C_{+}+\frac{1}{12} S & r_{0} \\
\hline r_{0} & C_{-}+\frac{1}{12} S
\end{array}\right) .
$$

Here $C_{ \pm}$are the SD and ASD parts of the Weyl tensor, $r_{0}$ is the trace-free Ricci curvature, and $S$ is the scalar curvature which acts by scalar multiplication. We are now ready to present the three systems of PDEs 
4.1. Self-dual null-Kähler. The condition $C_{-}=0$ is equivalent to

$$
\frac{\partial^{4} \Theta}{\partial y^{i} \partial y^{j} \partial y^{k} \partial y^{l}}=0 .
$$

Therefore the most general self-dual null-Kähler metric in four dimensions is of the form (1.1) with

$$
\Theta=\sum_{i, j, k} \Gamma_{i j k} y^{i} y^{j} y^{k}
$$

where the functions $\Gamma_{i j k}$ depend only on $\left(x^{1}, x^{2}\right)$, as the coordinate freedom (3.7) can be used to remove the quadratic and linear terms from $\Theta$. The resulting metric is a Walker's projective extension [17] of a projective structures on the surface $M=\mathcal{X} / \mathcal{D}$.

4.2. Anti-self-dual null-Kähler. The condition $C_{+}=0$ is equivalent to a 4 th order PDE for $\Theta$ :

$$
\begin{aligned}
f & =\Theta_{x^{1} y^{2}}-\Theta_{x^{2} y^{1}}+\Theta_{y^{1} y^{1}} \Theta_{y^{2} y^{2}}-\left(\Theta_{y^{1} y^{2}}\right)^{2} \\
\Delta_{g} f & :=f_{x^{1} y^{2}}-f_{x^{2} y^{1}}+\Theta_{y^{2} y^{2}} f_{y^{1} y^{1}}+\Theta_{y^{1} y^{1}} f_{y^{2} y^{2}}-2 \Theta_{y^{1} y^{2}} f_{y^{1} y^{2}}=0
\end{aligned}
$$

where subscripts denote partial derivatives, i. e. $\Theta_{x^{1}}=\partial \Theta / \partial x^{1}$ etc. Note that $\Delta_{g}$ is the Laplace-Beltrami operator of the metric $g$, and the expression for $f$ agrees with the general formula (3.8). This equation is integrable by twistor transform [15], the dressing method [3], and the Manakov-Santini inverse scattering transform [39]. The general solution depends on 4 functions of 3 variables. It has recently been shown to arise from a second-order integrable Lagrangian [18].

4.3. Null-Kähler Einstein. As the scalar curvature of null-Kähler manifolds always vanishes, the Einstein condition is equivalent to the vanishing of the Ricci tensor of $g$. The resulting second order PDE (3.9) on $\Theta$ is the hyper-heavenly equation of Plebański and Robinson [30] for non-expanding metrics with self-dual Weyl tensor $C_{+}$of type $\mathbf{N}$. (Recall that $(\mathcal{X}, g)$ is called hyper-heavenly if the self-dual Weyl tensor is algebraically special, i. e. has a repeated root when regarded as a binary quartic. Type $\mathbf{N}$ corresponds to a repeated root of order 4).

4.4. Heavenly equation. Imposing the Einstein condition together with the anti-selfduality of the Weyl tensor reduces the 4th order Eq. (4.5) to a second order PDE

$$
f=0 \text {. }
$$

This is Plebański's second heavenly equation [29]. The resulting metric is pseudo-hyperKähler. 


\section{Anti-self-duality and Isomonodromy}

In this section we shall assume that $(\mathcal{X}, N, g)$ is a null-Kähler four-manifold with antiself-dual Weyl curvature which is cohomogeneity-one, i. e. there exists an isometry group $G$ acting transitively on three-dimensional surfaces in $\mathcal{X}$. The four-dimensional cohomogeneity-one metrics can be classified according to the Bianchi type of the threedimensional real Lie algebra of $G$. Locally $\mathcal{X}=\mathbb{R} \times G$ and the ASD cohomogeneity-one null-Kähler condition reduces to solving a system of ODEs. To write this system down, and recognise it as the isomonodromy problem for Painlevé I and II if $G=S L(2)$, we shall use the twistor methods [15,28]. We shall therefore work in the holomorphic category, and assume that $\mathcal{X}_{\mathbb{C}}$ is a complex oriented four-manifold, and $(N, g)$ are holomorphic.

Definition 5.1. An $\alpha$-surface is two-dimensional surface $\zeta \subset \mathcal{X}_{\mathbb{C}}$ such that for all $p \in$ $\mathcal{X}_{\mathbb{C}}$ the tangent space $T_{p} \xi$ is a totally null plane with self-dual tangent bi-vector.

The Nonlinear Graviton theorem of Penrose [28] states that there locally exist a threeparameter family of $\alpha$-surfaces iff the self-dual part of the Weyl tensor of $g$ vanishes. The twistor space $\mathcal{Y}$ of an ASD four-manifold is defined to be the space of $\alpha$-surfaces. It is a three-dimensional complex manifold with a four-parameter family of rational curves with normal bundle $\mathcal{O}(1) \oplus \mathcal{O}(1)$. The points of the three-dimensional twistor space $\mathcal{Y}$ are $\alpha$-surfaces in $\mathcal{X}_{\mathbb{C}}$ : There is a rational curve $L_{p} \cong \mathbb{C P}^{1}$ worth of such surfaces through each point $p \in \mathcal{X}_{\mathbb{C}}$, and therefore points in $\mathcal{X}_{\mathbb{C}}$ correspond to rational curves in $\mathcal{Y}$. The conformal structure on $\mathcal{X}_{\mathbb{C}}$ is defined by declaring two points $p_{1}, p_{2} \in \mathcal{X}_{\mathbb{C}}$ to be null-separated iff the corresponding rational curves in $\mathcal{Y}$ intersect at one point.

The correspondence between $\mathcal{X}_{\mathbb{C}}$ and $\mathcal{Y}$ can be expressed in the the double fibration picture (see e.g. [26]).

$$
\mathcal{X}_{\mathbb{C}} \stackrel{r}{\longleftarrow} \mathcal{F} \stackrel{q}{\longrightarrow} \mathcal{Y},
$$

where the five-complex-dimensional correspondence is defined by

$$
\mathcal{F}=\mathcal{Y} \times\left.\mathcal{X}_{\mathbb{C}}\right|_{\zeta \in L_{p}}=\mathcal{X}_{\mathbb{C}} \times \mathbb{C P}^{1}
$$

where $L_{p}$ is the rational curve in $\mathcal{Y}$ that corresponds to $p \in X_{\mathbb{C}}$, and $\zeta \in \mathcal{Y}$ lies on $L_{p}$. The twistor space arises as a quotient of $\mathcal{F}$ by a two-dimensional integrable distribution spanned by the vector fields

$$
l_{1}=E_{11}-\lambda E_{12}+f_{1} \frac{\partial}{\partial \lambda}, \quad l_{2}=E_{21}-\lambda E_{22}+f_{2} \frac{\partial}{\partial \lambda},
$$

where $\lambda$ is an affine coordinate on $\mathbb{C P}^{1}$, the functions $f_{1}, f_{2}$ on $\mathcal{F}$ are cubic in $\lambda$, and $E_{i j}$ are four independent holomorphic vector fields on $\mathcal{X}_{\mathbb{C}}$ such that the conformal structure defined by the contravariant metric

$$
g=\frac{1}{2}\left(E_{11} \otimes E_{22}+E_{22} \otimes E_{11}-E_{12} \otimes E_{21}-E_{21} \otimes E_{12}\right) .
$$

The Frobenius integrability condition

$$
\left[l_{1}, l_{2}\right]=0 \quad\left(\bmod l_{1}, l_{2}\right)
$$

is equivalent to the anti-self-duality condition $C_{+}=0$ on $\mathcal{X}_{\mathbb{C}}$. If the integrability condition holds then there is a $\mathbb{C P}^{1}$-worth of $\alpha$-surfaces spanned by $\left\{E_{11}-\lambda E_{12}, E_{21}-\lambda E_{22}\right\}$ 
through any point in $\mathcal{X}_{\mathbb{C}}$. If all vectors $E_{i j}$ are real then the signature of $g$ is $(2,2)$, and there exists an $\mathbb{R P}^{1}$-worth of real $\alpha$-surfaces through each point of a real four-manifold $\mathcal{X}$. The null-Kähler condition on top of anti-self-duality gives rise to an additional structure on the twistor space:

\section{Theorem 5.2. [15] Let $\mathcal{Y}$ be a three-dimensional complex manifold with}

(1) A four-parameter family of rational curves with normal bundle $\mathcal{O}(1) \oplus \mathcal{O}(1)$.

(2) A preferred section of $\kappa^{-1 / 4}$ where $\kappa$ is the holomorphic canonical bundle of $\mathcal{Y}$.

(3) An anti-holomorphic involution $\rho: \mathcal{Y} \rightarrow \mathcal{Y}$ fixing a real equator of each rational curve, and leaving the section of $\kappa$ above invariant.

Then the real moduli space $\mathcal{X}$ of the $\rho$-invariant curves is equipped with a restricted conformal class $[\mathrm{g}]$ of anti-self-dual null-Kähler metrics: if $g \in[\mathrm{g}]$, and $\Omega$ is a nullKähler two-form, then $\left(\hat{g}=F^{2} g, \hat{\Omega}=F^{3} \Omega\right)$ is also null-Kähler for any function $F$ such that $d F \wedge \Omega=0$.

Conversely, given a real analytic ASD null-Kähler metric, there exists a corresponding twistor space $\mathcal{Y}$ with the above structures.

If one is only interested in the complexified picture, where $g$ and $N$ are holomorphic on $\mathcal{X}_{\mathbb{C}}$, then condition (3) in Theorem 5.2 can be dropped.

From now on we shall additionally assume that there exists a three-dimensional complex Lie group $G$ acting on $\mathcal{X}_{\mathbb{C}}=\mathbb{C} \times G$ by isometries with generically threedimensional orbits. We shall make a choice for $G$, and take it to be $S L(2, \mathbb{C})$ (or $S L(2, \mathbb{R}$ ) if $\mathcal{X}$ is a real four-manifold with a $(2,2)$ metric). Its Lie algebra is generated by the left invariant vector fields $L_{1}, L_{2}, L_{3}$ on $G$ which satisfy

$$
\left[L_{1}, L_{2}\right]=L_{2}, \quad\left[L_{1}, L_{3}\right]=-L_{3}, \quad\left[L_{2}, L_{3}\right]=2 L_{1} \text {. }
$$

The conformal isometries are generated by the right-invariant vector fields $R_{\alpha}, \alpha=$ $1,2,3$ on $G$. The metric on $\mathcal{X}_{\mathbb{C}}$ will be expressed in terms of the left-invariant one-forms $\sigma^{1}, \sigma^{2}, \sigma^{3}$ on $S L(2)$ such that

$$
\left.\mathcal{L}_{R_{\alpha}} \sigma^{\beta}=0, \quad L_{\alpha}\right\lrcorner \sigma^{\beta}=\delta_{\alpha}^{\beta}
$$

and

$$
d \sigma^{1}=2 \sigma^{3} \wedge \sigma^{2}, \quad d \sigma^{2}=\sigma^{2} \wedge \sigma^{1}, \quad d \sigma^{3}=\sigma^{1} \wedge \sigma^{3} .
$$

The $G$-action on $\mathcal{X}_{\mathbb{C}}$ maps $\alpha$-surfaces to $\alpha$-surfaces and thus gives rise to a holomorphic group action of $G$ on the twistor space $\mathcal{Y}$. Let the $\widetilde{R}_{\alpha}, \alpha=1,2,3$ be holomorphic vector fields on $\mathcal{Y}$ generating this action and corresponding to $R_{\alpha}$. Consider a quartic

$$
s=\operatorname{vol} \mathcal{Y}\left(\widetilde{R}_{1}, \widetilde{R}_{2}, \widetilde{R}_{3}\right),
$$

where voly is a holomorphic volume form on the twistor space with values in $\mathcal{O}(4)$. This quartic vanishes at each twistor line at four points, where the holomorphic vector fields corresponding to the isometries become linearly dependent. We shall, form now on assume that the four zeros of the quartic coincide, and so $s$ gives a preferred section of $\kappa^{-1 / 4}$. The corresponding conformal structure therefore contains a null-Kähler structure by Theorem 5.2. We shall first need to establish two technical results about the quartic (5.7), as the canonical form of the metric depends on whether $s$ vanishes identically, or not. 
Proposition 5.3. If the quartic (5.7) vanishes identically then the conformal class containing $g$ is hyper-complex, or equivalently if there exists a holomorphic fibration of $\mathcal{Y}$ over $\mathbb{C P}^{1}$ such that the twistor curves are sections of this fibration.

Proof. We shall first introduce some notation. Let $\pi^{i}=\left[\pi^{1}, \pi^{2}\right]$ be homogeneous coordinates on $\mathbb{C} \mathbb{P}^{1}$-fibres of the bundle $\mathbb{P}\left(\mathbb{S}_{+}\right)$such that $\lambda=-\pi^{2} / \pi^{1}$ in the patch where $\pi^{1} \neq 0$, and let $\pi_{i}=\sum_{j} \varepsilon_{j i} \pi^{j}$. Assemble the frame in (5.2) into a vector-valued two by two matrix $E$ with components $E_{i j}$ so that the twistor distribution $\mathcal{D} \mathcal{Y} \equiv \mathcal{O}(-1) \otimes \mathbb{C}^{2}$ given by (5.2) takes the form

$$
l_{i}=\sum_{j} \pi^{j} E_{i j}+f_{i} \frac{\partial}{\partial \lambda}, \quad i=1,2 .
$$

For this to be homogeneous of degree 1 in $\pi$ the functions $\left(f_{1}, f_{2}\right)$ need to be sections of $\mathcal{O}(3)$. Let $e^{i j}$ be a frame of one-forms dual to $E_{i j}$ so that $\left.E_{i j}\right\lrcorner e^{m n}=\delta_{i}{ }^{m} \delta_{j}{ }^{n}$, and the metric is given by

$$
g=\frac{1}{2}\left(e^{11} \otimes e^{22}+e^{22} \otimes e^{11}-e^{12} \otimes e^{21}-e^{21} \otimes e^{12}\right) .
$$

In the double fibration picture (5.1) the quartic $s$ pulls back to a quartic on $\mathcal{F}$ given by

$$
q^{*}(s)=(d \lambda \wedge \mathrm{vol})\left(l_{1}, l_{2}, R_{1}, R_{2}, R_{3}\right),
$$

where vol is the holomorphic volume form on $\mathcal{X}_{\mathbb{C}}$ such that vol $\left(E_{11}, E_{21}, E_{12}, E_{22}\right)=$ 1 , and we have chosen to work in an invariant frame, where the lifts of $R_{\alpha}$ s to the correspondence space $\mathcal{F}$ are given by $R_{\alpha} \mathrm{s}$. Such a frame always exists, as given a cohomogeneity-one metric of the form (5.3) we can choose a frame of one-forms $e^{i j}$ which are linear combinations of the left invariant one-forms on on $G$, and $d t$. Here $t$ is a function on such that the surfaces of constant $t$ are the orbits of $S L(2)$, and so $d t$ is normal to the surfaces of homogeneity. The coefficients of this combination only depend on $t$, so the self-dual two-forms

$$
e^{11} \wedge e^{21}, \quad e^{11} \wedge e^{22}-e^{21} \wedge e^{12}, \quad e^{12} \wedge e^{22}
$$

constructed from the frame $e^{i j}$ are also $G$-invariant. Therefore the lift of the $S L(2)$ action to the bundle $\mathbb{S}_{+}$is trivial, but the correspondence space is the projectivisation of this bundle.

Define $T_{i j}$ by

$$
d t=\sum_{i j} T_{i j} e^{i j}, \quad \text { so that } T_{i j}=E_{i j}(t) .
$$

where we have used $d=\sum_{i, j} e^{i j} \otimes E_{i j}$. Let vol $=d t \wedge \operatorname{vol}_{S L(2)}$, so that

$$
\operatorname{vol}_{S L(2)}\left(R_{1}, R_{2}, R_{3}\right)=1 \text {. }
$$

Therefore, using (5.9),

$$
q^{*}(s)=\frac{1}{2} \sum_{i, j, m, n} \varepsilon_{-}^{m n} T_{i j} d \lambda \wedge e^{i j}\left(l_{m}, l_{n}\right)
$$


where $\varepsilon_{-}$is the symplectic structure on $\mathbb{S}_{-}{ }^{*}$. Using $e^{i j}\left(l_{m}, \cdot\right)=\delta^{i}{ }_{m} \pi^{j}$ gives

$$
\begin{aligned}
q^{*}(s) & =\sum_{i, j, k, m, n} T_{i j} f_{m} \pi^{k} \delta^{i}{ }_{n} \delta^{j}{ }_{k} \varepsilon_{-}{ }^{m n} \\
& =\sum_{i, j, k} f_{i} T_{j k} \pi^{k} \varepsilon_{-}{ }^{i j} .
\end{aligned}
$$

If the invariant frame $E_{i j}$ is also such that $f_{1}=f_{2}=0$, then $q^{*}(s)$ given by (5.10) is identically zero. In [13] it was shown that a frame with $f_{1}=f_{2}=0$ (and therefore a holomorphic fibration $\mathcal{Y} \rightarrow \mathbb{C P}^{1}[4,11,21]$ ) exists iff $g$ is hyper-complex. Therefore the hyper-complex condition is necessary for the vanishing of $s$.

\section{Remarks}

\section{1}

A complexified hyper-Hermitian (which in four dimensions is equivalent to complexified hyper-complex) structure on $\mathcal{X}_{\mathbb{C}}$ is a triple of holomoprhic Hermitian endomorphisms $I, J, K$ of $T \mathcal{X}_{\mathbb{C}}$ which satisfy the algebra of quaternions. If $J=i S, K=-i T$, and $I, S, T$ are all real, then they form a pseudo-hyper-Hermitian structure on split-signature real four-manifold $\mathcal{X}$. The endomorphism $I$ endows $\mathcal{X}$ with the structure of a twodimensional complex Kähler manifold, and so does every other complex structure $a I+$ $b S+c T$ parametrised by the points of the hyperboloid

$$
a^{2}-b^{2}-c^{2}=1
$$

\section{2}

The converse of Proposition 5.3 does not hold: if $g$ is ASD and Ricci-flat (and therefore hyper-complex) but the $S L(2)$ action rotates the covariantly constant self-dual two forms, then $s$ does not vanish. That is to say the covariantly constant frame does not have to be invariant. The basis of two-forms in an invariant frame (which, as we have argued, always exists) is not covariantly constant and so $f_{1}$ and $f_{2}$ will not vanish. ASD TaubNUT or the Atiyah-Hitchin metrics are both examples illustrating this phenomenon. We can however say more if the isometric group action preserves the null-Kähler structure:

Lemma 5.4. If $\left(\mathcal{X}_{\mathbb{C}}, g, N\right)$ is a cohomogeneity-one $S L(2)$ invariant null-Kähler structure which is Ricciflat, and such that $N$ is preserved by the group action, then the quartic $s$ vanishes identically.

Proof. Let $\iota \in \Gamma\left(\mathbb{S}_{+}\right)$be the covariantly constant spinor defining $N$. Then $\iota$ must be in a linear combination of the covariantly constant basis of $\mathbb{S}_{+}$(which exists for ASD metrics iff they are Ricci flat) with constant coefficients (or it can not be parallel). Therefore the null structure $N$ belongs to the hyperboloid (5.11) of complex structures defined by the covariantly constant basis. The group $S L(2)$ acts on this hyperboloid, and we require that it fixes $N$. But this implies that it must fix all other points of the hyperboloid (as otherwise the Lie algebra relations would be violated). Therefore the covariantly constant frame is also invariant and $s=0$. 


\section{3}

Proposition 5.3 was established by Hitchin who used representation-theoretic arguments [20] under an additional assumption that the twistor space admits a real structure which singles out a Riemannian real section of $\mathcal{X}_{\mathbb{C}}$. In these circumstances the quartic $s$ either vanishes identically, or it admits two repeated roots, or all four roots are distinct. This assumption is not valid in the context of null-Kähler structures and split signature metrics.

Lemma 5.5. If $\left(\mathcal{X}_{\mathbb{C}}, g\right)$ is hyper-Hermitian, and null-Kähler, then the metric $g$ is conformal to a Ricci-flat metric.

Proof. We shall use the formulation of the hyper-Hermitian condition due to Boyer [4], which is also applicable in the complexified setting [13]: a metric on $\mathcal{X}_{\mathbb{C}}$ is hyperHermitian if and only if there exists a basis $\left(\Sigma^{1}, \Sigma^{2}, \Sigma^{2}\right)$ of $\Lambda^{2}{ }_{+}$such that

$$
d \Sigma^{\alpha}=2 A \wedge \Sigma^{\alpha}, \quad \alpha=1,2,3
$$

for some one-form $A$. Moreover a hyper-Hermitian $g$ is locally conformal to Ricci-flat iff $A$ closed. The formula (5.12) together with the isomorphism (4.3) imply the existence of a basis $(o, \rho)$ of $\Gamma\left(\mathbb{S}_{+}\right)$such that

$$
\nabla o=A \otimes o, \quad \nabla \rho=A \otimes \rho .
$$

Let $\iota \in \Gamma\left(\mathbb{S}_{+}\right)$be the covariantly constant spinor defining $N$. Then $\iota=h_{1} o+h_{2} \rho$ for some functions $h_{1}, h_{2}$ on $\mathcal{X}_{\mathbb{C}}$. But then

$$
\nabla\left(h_{1} o+h_{2} \rho\right)=0
$$

gives $h_{1}=$ const $\cdot h_{2}$ and $A=-d \ln \left(h_{1}\right)$ so $g$ is conformal to a Ricci-flat metric.

Proposition 5.6. Let $g$ be an SL(2)-invariant cohomogeneity-one metric with anti-selfdual Weyl tensor on $\mathcal{X}_{\mathbb{C}}$, such that the quartic (5.7) does not identically vanish. Then the quartic vanishes on each twistor line at one point to order 4 if and only if there exists a null-Kähler structure $N$ (compatible with some metric in the conformal class of $g$ ) which is Lie-derived by the SL(2) action.

Proof. First assume that the quartic $s$ vanishes to order 4. Therefore its pull-back (5.8) to $\mathcal{F}$ factories as

$$
q^{*}(s)=\left(\sum_{i} \iota_{i} \pi^{i}\right)^{4},
$$

where $[\pi]$ are homogeneous coordinates on the fibres of $\mathbb{P}\left(\mathbb{S}_{+}\right)$and $\iota$ is a section of $\mathbb{S}_{+}$. This is a pull-back from $\mathcal{Y}$, so it is constant along the twistor distribution (5.2), i. e.

$$
l_{i}\left(q^{*}(s)\right)=0, \quad i=1,2
$$

which implies that $\iota$ satisfies the rank-one conformally invariant twistor equation

$$
\left.\nabla_{i(j} \iota_{k}\right)=0,
$$

where $\nabla$ is the spin connection on $\mathbb{S}_{+}$induced by the Levi-Civita connection of $g$. In [15] it was shown that in this case there exists a conformal rescaling

$$
\hat{g}=F^{2} g, \quad \hat{\imath}=F \iota, \quad \hat{\varepsilon}_{+}=F \varepsilon_{+}
$$


such that $\hat{\imath}$ is parallel with respect to the Levi-Civita connection of $\hat{g}$. This section defines the null-Kähler structure via the isomorphism (4.3).

Conversely, let us assume that $N$ is an $S L(2)$-invariant null-Kähler structure. Then, by Theorem 5.2, $N$ gives rise to a divisor line bundle over $\mathcal{Y}$ given by a canonical section of $\kappa^{-1 / 4}$. The zero-set of this section pulls back to the hypersurface

$$
\Omega\left(l_{1}, l_{2}\right)^{1 / 2}=0
$$

in $\mathcal{F}$, where $\Omega$ is the fundamental two-form of $N$ given by (2.5), and $l_{1}, l_{2}$ span the twistor distribution. As $N$ is invariant under the $S L(2)$ action, the holomorphic vector fields $\widetilde{R}_{\alpha}, \alpha=1,2,3$ on $\mathcal{Y}$ preserve the canonical section of $\kappa^{-1 / 4}$, and so are tangent to the surface of vanishing (5.15), or equivalently

$$
\sum_{i} \pi_{i} \iota^{i}=0
$$

where $\iota$ is the section of $\mathbb{S}_{+}$corresponding, via (4.3), to $N$. Every twistor line intersects this surface at one point given, in homogeneous coordinates, by $[\pi]=[\iota]$. Therefore the quartic $s$ (which by assumption does not vanish identically) vanishes at this point to order 4.

We are now going to use the structure of the twistor distribution (5.2) to establish Theorem 1.2 from the introduction.

Proof of Theorem 1.2. Let $t: \mathcal{X}_{\mathbb{C}} \rightarrow \mathbb{C}$ be a function parametrising the orbits of $G=$ $S L(2)$ in $\mathcal{X}_{\mathbb{C}}$ such that

$$
\mathcal{L}_{R_{\alpha}} t=0, \quad \alpha=1,2,3 .
$$

We can choose coordinates on $G$ such that ${ }^{4}$

$$
g=\sum_{\alpha, \beta} \gamma_{\alpha \beta}(t) \sigma^{\alpha} \otimes \sigma^{\beta}+\sum_{\alpha} n_{\alpha}(t)\left(\sigma^{\alpha} \otimes d t+d t \otimes \sigma^{\alpha}\right),
$$

where $\gamma$ is a symmetric 3 by 3 matrix and $n$ is a vector with components depending on $t$.

We can express the frame $E_{i j}$ in the distribution (5.2) in terms of the vector field $\partial_{t}$, and three linearly independent vector fields $P, Q, R$ tangent to $G$ which are $t$-dependent and invariant under left translations. A convenient choice which gives rise to the general metric of the form (1.2) is

$$
E_{11}=Q, \quad E_{22}=P, \quad E_{12}=-2 \frac{\partial}{\partial t}, \quad E_{21}=2 \frac{\partial}{\partial t}-R .
$$

The invariance condition implies that the functions $f_{1}$ and $f_{2}$ in (5.2) are constant on $G$, and so depend only on $\lambda$ and $t$. The quartic $s$ is proportional to $\left(f_{1}-\lambda f_{2}\right)$. By Proposition (5.6) it must have a quadrupole zero which can be moved to $\lambda=\infty$ by a Möbius transformation. Using the freedom in the frame rotations of the frame we set $\left(f_{1}=-1, f_{2}=0\right)$ so that

$$
l_{1}=Q+2 \lambda \frac{\partial}{\partial t}-\frac{\partial}{\partial \lambda}, \quad l_{2}=2 \frac{\partial}{\partial t}-R-\lambda P .
$$

\footnotetext{
4 This form is general, but is different from the one usually used (see [20,33-35]), where the vector field $\partial / \partial t$ is not null, and orthogonal to the orbits of $G$. We shall explain the connection between the two forms (which are equivalent) in Sect. 5.
} 
Now consider a pair of linear combinations of $l_{1}$ and $l_{2}(5.2)$ given by

$$
\begin{aligned}
& m_{1}:=\frac{l_{1}-\lambda l_{2}}{f_{1}+\lambda f_{2}}=\frac{\partial}{\partial \lambda}-Q-\lambda R-\lambda^{2} P, \\
& m_{2}:=\frac{1}{2} \frac{f_{2} l_{1}-f_{1} l_{2}}{f_{1}+\lambda f_{2}}=\frac{\partial}{\partial t}-\frac{1}{2} R-\frac{1}{2} \lambda P .
\end{aligned}
$$

Since the conformal class is anti-self-dual, the integrability condition (5.4) implies that $\left[m_{1}, m_{2}\right]=0$, modulo $m_{1}$ and $m_{2}$. As the Lie bracket $\left[m_{1}, m_{2}\right]$ does not contain $\partial_{\lambda}$ or $\partial_{t}$, it must be identically zero which yields

$$
\dot{P}=0, \quad \dot{Q}=\frac{1}{2}[R, Q]+\frac{1}{2} P, \quad \dot{R}=\frac{1}{2}[P, Q],
$$

where $\dot{P}=d P / d t$ etc.

The system (5.18) underlies the isomonodromic problem with irregular singularity of order four. To make this transparent we shall use the representation of $\mathfrak{s l}(2)$ by 2 by 2 matrices rather than vector fields, and make the replacements

$$
L_{1} \rightarrow\left(\begin{array}{cc}
1 / 2 & 0 \\
0 & -1 / 2
\end{array}\right), \quad L_{2} \rightarrow\left(\begin{array}{ll}
0 & 1 \\
0 & 0
\end{array}\right), \quad L_{3} \rightarrow\left(\begin{array}{ll}
0 & 0 \\
1 & 0
\end{array}\right) .
$$

The associated the Lax pair (5.17) is the isomonodromic Lax pair for Painlevé II if $P$ is diagonalisable, and is gauge equivalent to the isomonodromic Lax pair for Painlevé I or leads to a solvable equation if $P$ is nilpotent. The system (5.18) underlies the isomonodromic problem with irregular singularity of order four. To set this problem up consider a $2 \times 2$ matrix

$$
\mathcal{A}(t, \lambda)=Q+\lambda R+\lambda^{2} P
$$

where $\lambda \in \mathbb{C P}^{1}$, and $P, Q, R$ are elements of a matrix Lie algebra $\mathfrak{g}=\mathfrak{s l}(2)$ which also depend on a parameter $t$. When $t$ is allowed to vary on the complex plane, the matrix fundamental solution $\Psi$ of the ODE

$$
\frac{d \Psi}{d \lambda}=\mathcal{A} \Psi
$$

depends on $\lambda$ and $t$. The monodromy around the pole of order four at $\lambda=\infty$ does not depend on $t$ if $\Psi$ satisfies [22]

$$
\frac{\partial \Psi}{\partial \lambda}-\mathcal{A} \Psi=0, \quad \frac{\partial \Psi}{\partial t}-\mathcal{B} \Psi=0, \quad \text { where } \quad \mathcal{B}:=\frac{1}{2} R+\frac{1}{2} \lambda P .
$$

The compatibility conditions

$$
\partial_{t} \mathcal{A}-\partial_{\lambda} \mathcal{B}+[\mathcal{A}, \mathcal{B}]=0
$$

for the overdetermined linear system (5.19) reduce to system of nonlinear matrix ODEs (5.18) for $(P, Q, R)$. We shall follow the seminal work of $[25,26]$-but make different gauge choices - to reduce this system further to a single ODE. There are three gauge equivalence classes to consider. The first two lead to Painlevé ODEs and the last one is completely solvable. 
- If $P$ is diagonalisable, and $\mathfrak{g}=\mathfrak{s l}(2, \mathbb{C})$, then without loss of generality we can take $[22,26]$

$$
P=2 L_{1}, \quad R=u L_{2}-2 \frac{z}{u} L_{3}, \quad Q=(2 z+t) L_{1}-u y L_{2}-\frac{2 y z+\frac{1}{2}-\alpha}{u} L_{3},
$$

where $u, y$ and $z$ are functions of $t$. Equations (5.18) become

$$
\dot{u}=-y u, \quad \dot{z}=-2 y z+\alpha-\frac{1}{2}, \quad \dot{y}=z+y^{2}+\frac{t}{2},
$$

which imply

$$
\ddot{y}=2 y^{3}+t y+\alpha,
$$

where $\alpha$ is a constant parameter. This is the Painlevé II equation.

- If $P$ is nilpotent, then (as it is also constant) we can chose it to be $L_{2}$. Assume that $\operatorname{Tr}(P R) \neq 0$, and perform a gauge transformation

$$
\mathcal{A} \rightarrow \gamma \mathcal{A} \gamma^{-1}+\partial_{\lambda} \gamma \cdot \gamma^{-1}, \quad \mathcal{B} \rightarrow \gamma \mathcal{B} \gamma^{-1}+\partial_{t} \gamma \cdot \gamma^{-1}
$$

with the group element $\gamma=\gamma(t)$ such that

$$
\partial_{t} \gamma \cdot \gamma^{-1}=y P, \quad \text { where } \quad y \equiv \frac{1}{8} \operatorname{Tr}\left(R^{2}\right) .
$$

Then

$$
P=L_{2}, \quad R=y L_{2}+4 L_{3}, \quad Q=-2 z L_{1}+\left(y^{2}+\frac{t}{2}\right) L_{2}-4 y L_{3}
$$

and

$$
\mathcal{B}=\frac{\partial}{\partial t}-\frac{1}{2}\left(R+y L_{2}\right)-\frac{1}{2} \lambda P .
$$

The compatibility conditions (5.20) give

$$
\dot{y}=z, \quad \dot{z}=6 y^{2}+t \quad \text { so that } \ddot{y}=6 y^{2}+t
$$

which is the Painlevé I equation.

- Finally let us consider the case where $P$ is nilpotent, and $\operatorname{Tr}(P R)=0$. We shall set $P=L_{2}$, as in the case leading to (5.22), and consider

$$
R=\sum_{\alpha} r_{\alpha}(t) L_{\alpha}, \quad Q=\sum_{\alpha} q_{\alpha}(t) L_{\alpha}, \quad r_{3}=\operatorname{Tr}(P R)=0
$$

for some functions $r_{\alpha}, q_{\alpha}, \alpha=1,2,3$ of $t$. The 3 rd equation in (5.18) gives

$$
q_{1}=-2 \dot{r}_{2}, \quad \dot{r}_{1}=q_{3} .
$$

The 2 nd equation in (5.18) gives

$$
2 \ddot{r}_{1}+\dot{r}_{1} r_{1}=0, \quad 2 \ddot{r}_{2}+\dot{r}_{1} r_{2}=0, \quad 2 \dot{q}_{2}-2 r_{2} \dot{r}_{2}-q_{2} r_{1}-1=0 .
$$

The first of these equations has a singular solution $r_{1}=$ const which eventually leads to a degenerate tetrad (5.16). We therefore focus on the regular solution, and absorb 
two constants of integrations in $r_{1}$ into an affine transformation of $t$ which results in a constant rescaling of the metric. The remaining two equations can also be solved:

$$
\begin{aligned}
r_{1} & =4 \tanh (t), \quad r_{2}=(a+b t) r_{1}-4 b, \\
q_{2} & =\frac{1}{4} \sinh (2 t)-\frac{d}{d t}\left((a+b t) r_{2}\right)+c \cdot \cosh (t)^{2},
\end{aligned}
$$

where $a, b, c$ are the remaining constants of integration.

Now we shall construct the conformal classes corresponding to Painlevé I and Painlevé II equations, and in each case find a null-Kähler metric in the conformal class. These structures will be expressed in terms of left-invariant one-forms (5.6). Each conformal class is represented by a covariant metric dual to (5.3)

$$
\frac{1}{2}\left(e^{11} \otimes e^{22}+e^{22} \otimes e^{11}-e^{12} \otimes e^{21}-e^{21} \otimes e^{12}\right) .
$$

The null-Kähler two-form $\Omega$ can be read off from the divisor quartic (5.7). In the spinorform

$$
\Omega=\iota \otimes \iota \otimes \varepsilon_{-},
$$

where $\iota \in \Gamma\left(\mathbb{S}_{+}{ }^{*}\right)$ is the parallel spinor. When regarded as a section of $\mathbb{P}\left(\mathbb{S}_{+}{ }^{*}\right)$ it gives a point on $\mathbb{C P}^{1}$ which is the quadruple root of the quartic (5.7). In our case this gives $\Omega$ proportional to $e^{11} \wedge e^{21}$. The proportionality factor will be found together with the conformal factor for the metric which makes $\Omega$ parallel.

\section{4}

For Painlevé II the one-forms dual to the tetrad (5.16) are

$$
\begin{aligned}
e^{22} & =\frac{1}{2} \sigma^{1}+\frac{z(2 z+t)}{u(4 y z+1-2 \alpha)} \sigma^{2}+\frac{u\left(z+\frac{t}{2}\right)}{4 y z+1-2 \alpha} \sigma^{3}, \\
e^{11} & =-\frac{2 z}{u(4 y z+1-2 \alpha)} \sigma^{2}-\frac{u}{4 y z+1-2 \alpha} \sigma^{3}, \\
e^{21} & =-\frac{2 y z+1-2 \alpha}{u(4 y z+1-2 \alpha)} \sigma^{2}+\frac{y u}{4 y z+1-2 \alpha} \sigma^{3}, \\
e^{12} & =-\frac{1}{2} d t-\frac{2 y z+1-2 \alpha}{u(4 y z+1-2 \alpha)} \sigma^{2}+\frac{y u}{4 y z+1-2 \alpha} \sigma^{3} .
\end{aligned}
$$

The unique conformal factor which makes the null-Kähler two-from parallel is

$$
k=4 y z+1-2 \alpha
$$

so that

$$
\Omega=2 \sigma^{3} \wedge \sigma^{2}
$$

and the metric is given by (1.2) with

$$
\gamma=\left(\begin{array}{ccc}
0 & \frac{z}{u} & \frac{u}{2} \\
\frac{z}{u} & \frac{8 z^{3}+\left(8 y^{2}+4 t\right) z^{2}+(8-16 \alpha) y z+8\left(\alpha-\frac{1}{2}\right)^{3}}{k u^{2}} & -2 \frac{2 y^{2} z+(1-2 \alpha) y-z(2 z+t)}{k} \\
\frac{u}{2} & -2 \frac{2 y^{2} z+(1-2 \alpha) y-z(2 z+t)}{k} & \frac{u^{2}\left(2 y^{2}+2 z+t\right)}{k}
\end{array}\right),
$$




$$
n=\left(0, \frac{2 y z-2 \alpha+1}{2 u},-\frac{y u}{2}\right) .
$$

The Weyl tensor is ASD if Painlevé II holds.

\section{5}

In the case of Painlevé I, we first read-off the tetrad form the form of $\mathcal{A}$ and $\mathcal{B}$ to be

$$
E_{11}=Q, \quad E_{22}=P, \quad E_{12}=-2 \frac{\partial}{\partial t}+y L_{2}, \quad E_{21}=2 \frac{\partial}{\partial t}-\left(R+y L_{2}\right) .
$$

Computing the dual tetrad gives

$$
\begin{aligned}
e^{22}= & \frac{y^{2}+\frac{t}{4}}{z} \sigma^{1}+\sigma^{2} \\
& -\frac{y}{4} \sigma^{3}+\frac{y}{2} d t, \\
e^{11}= & -\frac{1}{2 z} \sigma^{1}, \\
e^{21}= & \frac{y}{2 z} \sigma^{1}-\frac{1}{4} \sigma^{3}, \\
e^{12}= & \frac{y}{2 z} \sigma^{1}-\frac{1}{4} \sigma^{3}-\frac{1}{2} d t
\end{aligned}
$$

and rescalling the resulting metric and two-form by $k=16 z(t)$ gives

$$
\Omega=2 \sigma^{3} \wedge \sigma^{1}
$$

This is the only scaling factor which makes $\Omega$ closed. Using the same conformal factor for the metric yields and $g$ in the form (1.2) with

$$
\gamma=-\left(\begin{array}{ccc}
\frac{12 y^{2}+2 t}{z} & 4 & -3 y \\
4 & 0 & 0 \\
-3 y & 0 & z
\end{array}\right), \quad n=(0,0,-z)
$$

The two-form $\Omega$ is now parallel, as

$$
\nabla \Omega=\left(\frac{6 y^{2}+t-\dot{z}}{z} \sigma^{1}+\frac{3(z-\dot{y})}{z} \sigma^{2}\right) \otimes \Omega=0
$$

where we used (5.22). The null-Kähler structure is given by

$$
N=\frac{1}{2}\left(\sigma^{3} \otimes L_{2}-\frac{2}{z} \sigma^{1} \otimes \frac{\partial}{\partial t}\right) .
$$

Computing the self-dual part of the Weyl tensor we find that it vanishes as a consequence of Painlevé I 


\section{6}

Computing the dual tetrad in the case (5.23) gives

$$
\begin{aligned}
e^{22}= & (b \operatorname{coth}(t)-(a+b t)) \sigma^{1}+\sigma^{2} \\
& +\left(2 b(a+b t) \operatorname{coth}(t)+b^{2} \cosh (t)^{2}-\frac{1}{8} \sinh (t) \cosh (t)^{3}-(a+b t)^{2}-\frac{c}{4} \cosh (t)^{2}\right) \sigma^{3}, \\
e^{11}= & \frac{1}{4} \cosh (t)^{2} \sigma^{3}, \\
e^{21}= & -\frac{1}{4} \operatorname{coth}(t) \sigma^{1}-\frac{1}{2}\left(b \cosh (t)^{2}+(a+b t) \operatorname{coth}(t)\right) \sigma^{3}, \\
e^{12}= & -\frac{1}{2} d t-\frac{1}{4} \operatorname{coth}(t) \sigma^{1}-\frac{1}{2}\left(b \cosh (t)^{2}+(a+b t) \operatorname{coth}(t)\right) \sigma^{3}
\end{aligned}
$$

and rescaling the resulting metric and two-form by $k=8 \sinh (t) / \cosh (t)^{3}$ gives

$$
\Omega=\sigma^{3} \wedge \sigma^{1}
$$

This is the only scaling factor which makes $\Omega$ closed. Using the same conformal factor for the metric yields $g$ in the form (1.2) with

$$
\begin{aligned}
& \gamma=\left(\begin{array}{ccc}
\frac{2}{\sinh 2 t} & 0 & (a+b t) \operatorname{coth}(2 t) \\
0 & 0 & -\tanh (2 t) \\
(a+b t) \operatorname{coth}(2 t) & -\tanh (2 t) & 4(a+b t)^{2} \operatorname{coth}(t)+\frac{1}{8} \sinh (2 t)^{2}(1+2 c \cdot \operatorname{coth}(t))
\end{array}\right), \\
& n=\left(\cosh (t)^{-2}, 0,2(a+b t) \cosh (t)^{-2}+2 b \tanh (t)\right) .
\end{aligned}
$$

The two-form $\Omega$ is parallel and the Weyl tensor is anti-self-dual. The Ricci-tensor is

$$
r=\frac{1}{2} \sinh (t) \cosh (t)^{3} \sigma^{3} \otimes \sigma^{3} .
$$

\section{Remarks}

\section{7}

All solutions to PI are transcendental, and PII admits solutions expressible in terms of know functions only for integer and half-integer values of the parameter $\alpha$. These will lead to explicit metrics. On the other hand all metrics arising from (5.28) are explicit. The simplest is obtained by setting $a=b=c=0$. Setting $\tau=\tanh (t)$ gives

$$
\begin{aligned}
g= & \frac{1-\tau^{2}}{\tau} \sigma^{1} \otimes \sigma^{1}+\sigma^{1} \otimes d \tau+d \tau \otimes \sigma^{1}-2 \tau\left(\sigma^{2} \otimes \sigma^{3}+\sigma^{3} \otimes \sigma^{2}\right) \\
& +\frac{\tau^{2}}{2\left(1-\tau^{2}\right)^{2}} \sigma^{3} \otimes \sigma^{3} .
\end{aligned}
$$




\section{8}

The null-Kahler structures arising from PI and and PII can be distinguished by examining the restriction of kernel of the endomorphism $N$ to the orbits of $S L(2)$. In the PI case, this kernel-when regarded as the element of the Lie algebra of $S L(2)$ is nilpotent, but in the PII case it is not. This can be seen directly from (5.26) and (5.24). The kernel of $N$ spans a 2-parameter of $\alpha$-surfaces in $\mathcal{X}_{\mathbb{C}}$ which, at the twistor level, corresponds to a divisor hypersurface $\mathcal{N} \subset \mathcal{Y}$ which meets each twistor line to order 4 . This hypersurface is preserved by the $S L(2, \mathbb{C})$ action on the twistor space, so $\exists \tau \in \mathfrak{s l}(2)$ s. t. $\phi(\tau)=0$ where $\phi$ is the vector bundle homomorphism [20]

$$
\phi: \mathfrak{s l}(2, \mathbb{C}) \times \mathcal{Y} \rightarrow T \mathcal{Y} .
$$

The element $\tau$ is nilpotent in the case of Painlevé I and the solvable example, and semisimple in the case of Painlevé II.

\section{9}

The usual form of cohomogeneity-one metrics [20,33-35] is

$$
g=\frac{1}{4} d t^{2}+\sum_{\alpha, \beta} h_{\alpha \beta}(t)\left(\sigma^{\alpha} \otimes \sigma^{\beta}+\sigma^{\beta} \otimes \sigma^{\alpha}\right) .
$$

This arises from a frame of the form

$$
E_{11}=Q, \quad E_{22}=P, \quad E_{12}=-2 \frac{\partial}{\partial t}-\frac{1}{2} R, \quad E_{21}=2 \frac{\partial}{\partial t}-\frac{1}{2} R .
$$

Following the argument above which lead to the Lax pair (5.17) gives the system of ODEs [12]

$$
\dot{Q}=\frac{1}{4}[R, Q]+\frac{1}{2} P, \quad \dot{R}=\frac{1}{2}[P, Q], \quad \dot{P}=\frac{1}{4}[P, R]
$$

together with the isomonodromic Lax pair $\left[m_{1}, m_{2}\right]=0$, where

$$
m_{1}=-l_{1}+\lambda l_{2}=\partial_{\lambda}-\mathcal{A}, \quad m_{2}=\frac{1}{2} l_{2}=\partial_{t}-\mathcal{B}
$$

where now

$$
\mathcal{A}=Q+\lambda R+\lambda^{2} P, \quad \mathcal{B}=\frac{1}{4} R+\frac{1}{2} \lambda P .
$$

The gauge transformation

$$
\mathcal{A} \rightarrow \gamma \mathcal{A} \gamma^{-1}+\partial_{\lambda} \gamma \cdot \gamma^{-1}, \quad \mathcal{B} \rightarrow \gamma \mathcal{B} \gamma^{-1}+\partial_{t} \gamma \cdot \gamma^{-1}
$$

with $\gamma=\gamma(t)$ such that $\gamma^{-1} \cdot \dot{\gamma}=\frac{1}{4} R$ brings this Lax pair to the form (5.17), and the system (5.29) to the form (5.18). Indeed, we can verify that $\widetilde{P} \equiv \gamma \cdot P \cdot \gamma^{-1}$ is constant, and the other two equations also hold with $\widetilde{Q} \equiv \gamma \cdot Q \cdot \gamma^{-1}$ and $\widetilde{R} \equiv \gamma \cdot R \cdot \gamma^{-1}$. Thus the two forms of the metric are equivalent by a diffeomorphism. 


\subsection{0}

The isomonodromic Lax pair (5.17) arising as the combination of $\left(l_{1}, l_{2}\right)$ can be constructed invariantly using the notation from the proof of Proposition 5.3 as follows: Let $T$ be the vector field dual to $d t$ with respect to the isomorphism between tangent and cotangent bundle given by the metric. This vector is normal to the orbits of $S L(2)$, and is given by $T=\sum_{i, j} T^{i j} E_{i j}$ in the frame $E_{i j}$. First note that

$$
l_{i}(t)=\sum_{j} \pi^{j} T_{i j}, \quad \text { so that } \sum_{i, j} T^{i j} \pi_{j} l_{i}(t)=\sum_{i, j, k, m} \pi_{i} \pi_{j} \varepsilon_{k m} T^{k i} T^{m j}=0
$$

by symmetry. This implies that

$$
\sum_{i, j} T^{i j} \pi_{j} l_{i}=\sum_{i, j} T^{i j} \pi_{j} f_{i} \frac{\partial}{\partial \lambda}+\sum_{i, j, k} T^{i j} \pi_{j} \pi^{k} E_{i k}
$$

where the second term on the RHS is tangent to the orbits of $S L(2)$, so is in the span of the left-invariant vector fields and does not contain $\partial / \partial t$. Using (5.10) we identify the multiple of $\partial / \partial \lambda$ as the quartic $s$. Assuming that this quartic is not identically zero we define an $\mathcal{O}(-2)$-valued vector field

$$
m_{1}=\frac{\sum_{i, j} T^{i j} \pi_{j} l_{i}}{s}
$$

The second vector field $m_{2}$ does not contain the $\partial / \partial \lambda$ terms, and is defined by

$$
m_{2}=\frac{1}{2} \frac{\sum_{i, j} \varepsilon^{i j} f_{i} l_{j}}{s}
$$

This agrees with (5.17). If the metric is given by (1.2), then

$$
T=-\frac{1}{\sum_{\alpha, \beta} \gamma^{\alpha \beta} n_{\alpha} n_{\beta}}\left(\frac{\partial}{\partial t}-\sum_{\alpha, \beta} \gamma^{\alpha \beta} n_{\alpha} L_{\beta}\right)
$$

where $\gamma^{\alpha \beta}(t)$ is the inverse matrix of $\gamma_{\alpha \beta}(t)$.

\subsection{1}

Selecting a one-parameter family of transformations $\mathbb{R}^{*}$ in $S L(2, \mathbb{R})$ generated by a nonnull Killing vector $K$ reduces the null-Kähler ASD conditions to an Einstein-Weyl structure in $2+1$ dimension which additionally admits a parallel weighted null vector field. Such structures correspond to solutions of the dispersionless Kadomtsev-Petviashvili equation, and in [16], such solutions were constructed and shown to be constant on central quadrics and expressed in terms of solutions to Painlevé I or Painlevé II. 


\subsection{2}

If the Lie algebra $\mathfrak{g}$ underlying (5.18) is instead the Bianchi II algebra then the isomonodromic condition is the (derivative of ) the Airy equation (see [12], where a class of ASD null-Kähler four manifolds has been constructed). In [20,27] it was instead assumed that the quartic $s$ has four distinct zeros, and that $G=S L(2, \mathbb{C})$ which lead to the isomonodromic Lax pair [22] for Painlevé VI. If $s$ has two double zeroes then the conformal class contains an Einstein metric [20,34], and the isomonodromic Lax pair leads to Painlevé III.

Acknowledgements. I am grateful to Centro de Investigacion y de Estudios Avanzados in Mexico for the hospitality 19 years ago, when most of the results in Sect. 3 were obtained. My interest in the subject has been revived after I came across the works of Bridgeland [7,8], and Bridgeland and Strachan [9], where a nullKähler structure implicitly arises on the space of stability conditions on a Calabi-Yau three-fold tirangulated category. My work has been partially supported by STFC consolidated Grants ST/P000681/1, ST/T000694/1.

Open Access This article is licensed under a Creative Commons Attribution 4.0 International License, which permits use, sharing, adaptation, distribution and reproduction in any medium or format, as long as you give appropriate credit to the original author(s) and the source, provide a link to the Creative Commons licence, and indicate if changes were made. The images or other third party material in this article are included in the article's Creative Commons licence, unless indicated otherwise in a credit line to the material. If material is not included in the article's Creative Commons licence and your intended use is not permitted by statutory regulation or exceeds the permitted use, you will need to obtain permission directly from the copyright holder. To view a copy of this licence, visit http://creativecommons.org/licenses/by/4.0/.

Publisher's Note Springer Nature remains neutral with regard to jurisdictional claims in published maps and institutional affiliations.

\section{References}

1. Atiyah, M.F., Hitchin, N.J., Singer, I.M.: Self-duality in four-dimensional Riemannian geometry. Proc. R. Soc. Lond. Ser. A 362, 425-461 (1978)

2. Baum, H., Kath, I.: Parallel spinors and holonomy groups on pseudo-Riemannian spin manifolds. Ann. Glob. Anal. Geom. 17, 1-17 (1999)

3. Bogdanov, L.V., Dryuma, V.S., Manakov, S.V.: Dunajski generalization of the second heavenly equation: dressing method and the hierarchy. J. Phys. A Math. Theor. 40, 14383-14393 (2007)

4. Boyer, C.: A note on hyperhermitian four-manifolds. Proc. Am. Math. Soc. 102, 157-164 (1988)

5. Brozos-Vazquez, M., Garcia-Rio, E., Gilkey, P., Nikcevic, S., Vazquez-Lorenzo, R.: The Geometry of Walker Manifolds. Synthesis Lectures on Mathematics and Statistics, Morgan and Claypool (2009)

6. Bérard-Bergery, L., Ikemakhen, A.: Sur l'holonomie des variétés pseudo-Riemanniennes de signature $(n, n)$. Bull. Soc. Math. France 125, 93-114 (1997)

7. Bridgeland, T.: Geometry from Donaldson-Thomas invariants (2019). arXiv: 1912.06504

8. Bridgeland, T., Masoero, D.: On the monodromy of the deformed cubic oscillator (2020). arXiv:2006.10648

9. Bridgeland, T., Strachan, I.A.B.: Complex hyperkähler structures defined by Donaldson-Thomas invariants (2020). arXiv:2006.13059

10. Bryant, R.L.: Pseudo-Riemannian metrics with parallel spinor fields and vanishing Ricci tensor. In: Global Analysis and Harmonic Analysis, Seminar and Congress, vol. 4, pp. 53-94. Soc. Math. France, Paris (2000)

11. Calderbank, D.: Integrable background geometries. Sigma 10, 34 (2014)

12. Cole, M., Dunajski, M.: Twistor theory of the airy equation. SIGMA 10, 037 (2014). arXiv:1401.0025

13. Dunajski, M.: The twisted photon associated to hyper-hermitian four manifolds'. J. Geom. Phys. 30, 266-281 (1999)

14. Dunajski, M., Mason, L.J.: Twistor theory of hyper-Kähler metrics with hidden symmetries. J. Math. Phys. 44, 3430-3454 (2003)

15. Dunajski, M.: Anti-self-dual four-manifolds with a parallel real spinor. Proc. R. Soc. Lond. A 458, 12051222 (2002)

16. Dunajski, M., Tod, K.P.: Einstein-Weyl spaces and dispersionless Kadomtsev-Petviashvili equation from Painlevé I and II. Phys. Lett. A303, 253-264 (2002) 
17. Dunajski M., Tod, K. P.: Non-diagonal four-dimensional cohomogeneity-one Einstein metrics in various signatures Diff. Geom. Appl. 54, 11-30 (2017). arXiv:1609.02747

18. Ferapontov, E., Pavlov, M., Xue, L.: Second-order integrable Lagrangians and WDVV equations (2020). arXiv:2007.03768

19. Galaev, A., Leistner, T.: Recent developments in pseudo-Riemannian holonomy theory. IRMA Lect. Math. Theor. Phys. 16, 581-627 (2010)

20. Hitchin, N.J.: Twistor spaces, Einstein metrics and isomonodromic deformations. J. Differ. Geom. 42, 30-112 (1995)

21. Hitchin, N.J.: Hypercomplex manifolds and the space of framings. In: Hugget, et al. (eds.) The Geometric Universe. OUP (1998)

22. Jimbo, M., Miwa, T.: Monodromy preserving deformations of linear ordinary differential equations with rational coefficients. II. Physica 2D, 407-448 (1981)

23. Kobayashi, S., Nomizu, K.: Foundations of Differential Geometry, vol. 2. Interscience Publishers, Geneva (1969)

24. Lawson, H.B., Michelsohn, M.L.: Spin Geometry. Princeton Math. Series, vol. 38. Princeton University Press, Princeton (1989)

25. Mason, L.J., Woodhouse, N.M.J.: Self-duality and the Painlevé transcendents. Nonlinearity 6, 569 (1993)

26. Mason, L.J., Woodhouse, N.M.J.: Integrability. Self-duality and Twistor Theory. LMS Monograph, OUP, Oxford (1996)

27. Maszczyk, R., Mason, L.J., Woodhouse, N.M.J.: Selfdual Bianchi metrics and the Painlevé transcendents. Class. Quant. Grav. 11, 65-71 (1994)

28. Penrose, R.: Nonlinear gravitons and curved twistor theory. Gen. Rel. Grav. 7, 31-52 (1976)

29. Plebański, J.F.: Some solutions of complex Einstein equations. J. Math. Phys. 16, 2395-2402 (1975)

30. Plebański, J.F., Robinson, I.: Left-degenerate vacuum metrics. Phys. Rev. Lett. 37, 493-495 (1976)

31. Sparling, G.A., Tod, K.P.: An example of an H-space. J. Math. Phys. 22, 331-332 (1981)

32. Takasaki, K.: An infinite number of hidden variables in hyper-Kähler metrics. J. Math. Phys. 30, 1515 (1989)

33. Tod, K.P.: Self-dual Einstein metrics from the Painlevé VI. Phys. Lett. A 190, 221-224 (1994)

34. Tod, K.P.: Scalar-flat Kähler and hyper-Kähler metrics from Painlevé III. Class. Quantum Grav. 12, 1535-1547 (1995)

35. Tod, K.P.: Cohomogeneity-one metrics with self-dual Weyl tensor. In: Huggett, S. (ed.) Twistor Theory. Lect. Notes Pure Appl. Math., vol. 169. Marcel Dekker, New York (1995)

36. Walker, A.G.: Canonical form for a Riemannian space with a parallel field of null planes. Quart. J. Math. Oxf. 1, 69 (1950)

37. Yano, K., Davies, E.T.: Differential geometry on almost tangent manifolds. Ann. Mat. Pura Appl. 103, 131-160 (1975)

38. Yano, K., Ishihara, S.: Tangent and Cotangent Bundles: Differential Geometry. Dekker, New York (1973)

39. Yi, G., Santini, P.M.: The inverse spectral transform for the Dunajski hierarchy and some of its reductions, I: Cauchy problem and longtime behavior of solutions. J. Phys. A Math. Theor. 48, 215203 (2015)

Communicated by S. Gukov 